\title{
MONITORAMENTO DE PESTICIDAS E OUTROS POLUENTES ORGÂNICOS EM RECURSOS HÍDRICOS UTILIZANDO DISPOSITIVOS DE MEMBRANAS SEMIPERMEÁVEIS
}

\author{
FABRÍCIO VILELA PARREIRA * \\ JULIANA FELISBERTO ALVES ** \\ GILBERTO CALDEIRA BANDEIRA DE MELLO*** \\ WILLER HUDSON POS ${ }^{* \star * *}$ \\ ZENILDE G. G. VIOLA ***** \\ CIOMARA RABELO DE CARVALHO ******
}

\begin{abstract}
Efetuou-se revisão de trabalhos de pesquisa sobre dispositivos de membranas semipermeáveis (DMSP), contendo trioleína para monitoramento de ambientes aquáticos. Os DMSP têm sido utilizados como amostradores passivos de pesticidas e outros poluentes orgânicos lipofílicos em água. Esses dispositivos têm-se mostrado eficientes na amostragem de água para análise de substâncias em níveis de traços e ultra-traços, tais como pesticidas organoclorados, organofosforados, piretróides, bifenilas policloradas, hidrocarbonetos aromáticos policíclicos, dioxinas, furanos e outras substâncias orgânicas apolares. Atenção especial é dada aos modelos para cálculo da taxa de amostragem e concentração dos analitos nos corpos d'água. A tecnologia de DMSP continua a ser pesquisa por cientistas ambientais que buscam a otimização das condições para exposição e análise.
\end{abstract}

PALAVRAS-CHAVE: AMOSTRAGEM; DMSP; PESTICIDAS; ÁGUA.

Doutor em Química Analítica, Universidade Federal de Minas Gerais (UFMG), Centro de Tecnologia de Minas Gerais (CETEC), Setor de Medições Ambientais, Belo Horizonte, MG (e-mail: fabricio.vilela.parreira@cetec.br).

** Mestre em Saneamento, Meio Ambiente e Recursos Hídricos, UFMG, Escola de Engenharia, Departamento de Engenharia Sanitária e Ambiental (DESA), Belo Horizonte, MG (e-mail: julianafalves@yahoo.com.br).

*** Doutor em Ciências de Engenharia, Univ. Karlsruhe, Escola de Engenharia, DESA, Belo Horizonte, MG (e-mail: gilberto@desa.ufmg.br).

**** Doutor em Química Atmosférica, Georgia Institute of Technology, Instituto Mineiro de Gestão das Águas (IGAM), Belo Horizonte, MG (e-mail: whpos@anglogold.com.br).

**** Mestre em Saneamento, Meio Ambiente e Recursos Hídricos, UFMG, IGAM, Belo Horizonte, MG (e-mail: zenilde@igam.gov.br).

****** Mestre em Química Analítica, UFMG, CETEC, Setor de Medições Ambientais, Belo Horizonte, MG (e-mail: ciomara.rabelo@cetec.br). 


\section{INTRODUÇÃO}

Os pesticidas e outros poluentes orgânicos tóxicos representam grande problema pelo impacto potencial sobre a saúde humana e o meio ambiente. Em ecossistemas aquáticos, tais compostos são bioacumulados nos tecidos de espécies aquáticas devido sua baixa hidrossolubilidade. Para algumas espécies pode ocorrer também a biomagnificação, resultante da seqüência de etapas de bioacumulação que ocorrem ao longo da cadeia alimentar (BAIRD, 2002).

A análise qualitativa e quantitativa de contaminantes orgânicos hidrofóbicos em ecossistemas aquáticos tornou-se desafio contínuo para cientistas ambientais (VRANA et al., 2001). A amostragem desses poluentes em cursos d'água vem sendo realizada pela técnica convencional, mediante coleta de amostras em seções predefinidas de corpos d'água e sua preservação adequada para posterior análise em laboratório (ABNT, 1987ab). Todavia, esse tipo de amostragem apresenta algumas desvantagens em termos de custos e representatividade amostral, sendo necessário grande volume de amostras para a quantificação de poluentes em nível de traços. Além disso, retratam a condição de qualidade instantânea do ponto amostrado não fornecendo indicações sobre o desenvolvimento temporal das variáveis avaliadas. Assim, não permitem a detecção de episódios de contaminação no intervalo entre as amostragens (HUCKINS et al., 1999).

Os amostradores passivos têm sido utilizados para avaliar o comportamento dos compostos orgânicos em ambientes aquáticos por longo período (VRANA et al., 2001). A amostragem passiva, também conhecida como dosimetria passiva, consiste na coleta integrada de analitos em determinado período de amostragem, utilizando apenas pequeno dispositivo (KOT, ZABIEGATA e NAMIESNIK, 2000). Dentre as vantagens de utilização dessa técnica, além da exposta acima, está a redução no número de coletas e de análises, o que diminui o custo final de programas de monitoramento da qualidade das águas. Também ocorre a redução da decomposição da amostra pelo transporte e/ou armazenamento.

O amostrador passivo efetivo deve ser simples de fabricar e de manusear, pequeno o bastante para ser enviado ao laboratório, insensível aos interferentes e, sobretudo, sensível ao analito que se deseja coletar. Desta forma, alguns tipos de amostradores têm sido propostos, entre os quais dispositivos preenchidos com solvente, dispositivos com membrana semipermeável (DMSP), amostrador com extração e concentração no local, técnica de membrana líquida suportada e dispositivos preenchidos com fase sólida (KOT, ZABIEGATA e NAMIESNIK, 2000).

Os amostradores a base de DMSP têm se destacado como técnica inovadora na avaliação de diversos contaminantes orgânicos lipofílicos em vários campos de estudo como água, sedimentos/ solos e ar.

Este artigo apresenta uma revisão de trabalhos de pesquisa sobre dispositivos de membranas semipermeáveis (DMSP) contendo trioleína para monitoramento de pesticidas e outros poluentes orgânicos em ambientes aquáticos.

\section{AMOSTRADORES COM MEMBRANAS SEMIPERMEÁVEIS}

O amostrador com membranas semipermeáveis foi desenvolvido por Huckins, em 1990, para estudar a biodisponibilidade de compostos químicos hidrofóbicos para organismos aquáticos (HUCKINS, TUBERGEN e MANUWEERA, 1990). Consiste em membrana de polietileno (PE) de baixa densidade, fabricada sem aditivos e não-porosa, embora apresente cavidades transientes com diâmetro de aproximadamente $10 \AA$, formadas termicamente por movimentos randômicos das cadeias poliméricas. Os volumes livres das cavidades permitem a dissolução e difusão das moléculas orgânicas na membrana. Esse processo simula a difusão de contaminantes através das membranas de organismos vivos (PETTY et al., 1998). O interior da membrana é recheado com lipídio de alta massa molecular 
( $\geq 600 \mathrm{Da}$ ), normalmente trioleína (1,2,3-tri(cis-9-octadecenoil)glicerol) sintética de alta pureza (>95\%). Trioleína constitui o composto mais empregado por tratar-se de lipídio apolar encontrado em organismos aquáticos (Figura 1).

\section{FIGURA 1- AMOSTRADOR COM MEMBRANAS SEMIPERMEÁVEIS}

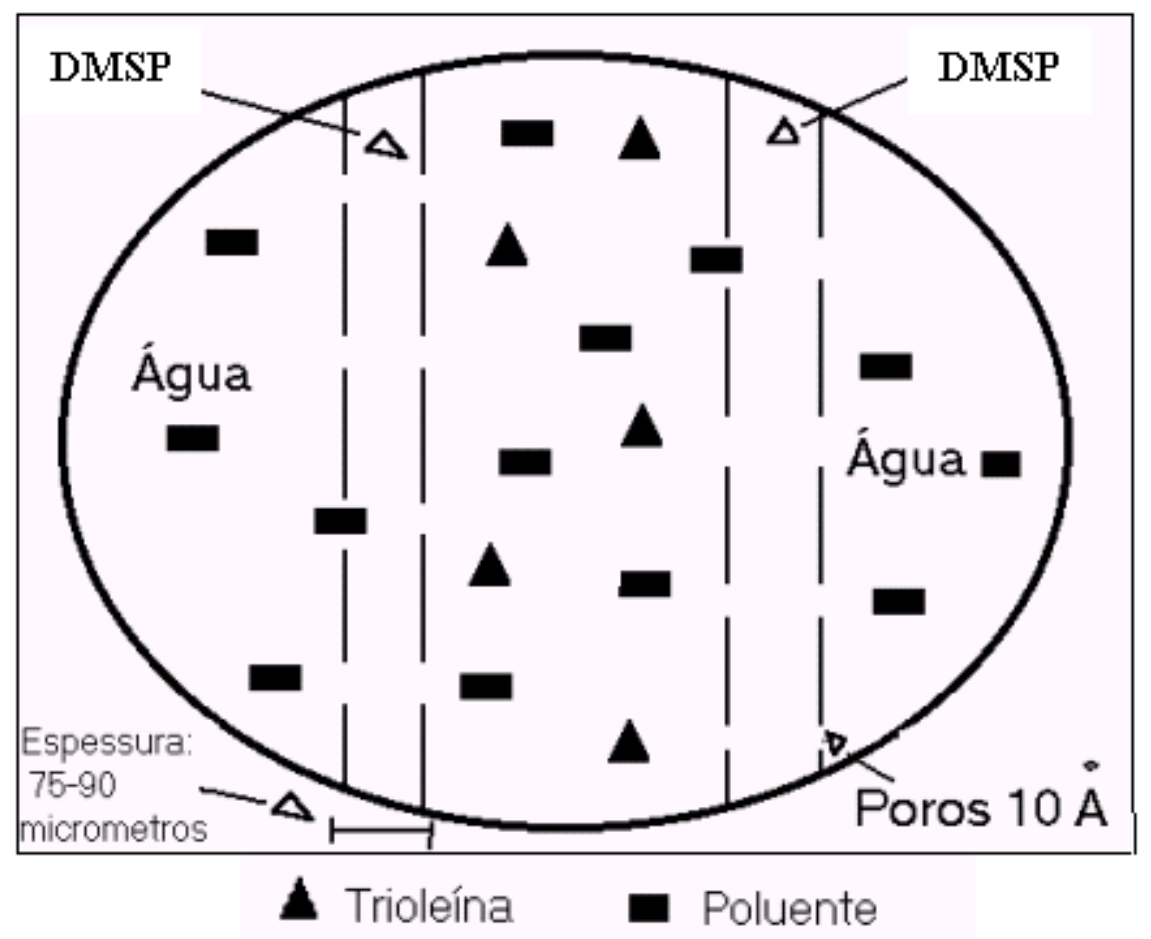

Fonte: Modificado de CHAPMAN (2002).

Segundo PETTY et al. (2000), a limitação do tamanho das cavidades do polietileno é importante para a seleção dos compostos. Geralmente apenas substâncias dissolvidas com massas molares menores que 600 Da são amostradas pelos dispositivos. Esse limite de massa molar é muito similar ao dos poros das biomembranas.

A capacidade de acúmulo de compostos hidrofóbicos por DMSP está relacionada com o coeficiente de partição octanol/água $\left(\mathrm{K}_{\text {ow }}\right)$ dos compostos. Aqueles com valores de $\mathrm{K}_{\text {ow }}$ mais altos têm maior possibilidade de serem concentrados nos dispositivos. Devido aos altos fatores de concentração alcançados pelos DMSP torna-se possível analisar níveis de contaminantes em ultra-traços (LEBO et al., 1995).

De acordo com PETTY et al. (2000), compostos orgânicos hidrofóbicos (com log $\mathrm{K}_{\mathrm{ow}} \geq 3$ ) são concentrados significativamente em níveis acima do ambiente. Qualquer composto com log $\mathrm{K}_{\mathrm{ow}} \geq 1$ pode ser concentrado pelo DMSP, entretanto seu uso não representa vantagem em relação aos outros procedimentos de amostragem para compostos com log $\mathrm{K}_{\mathrm{ow}}$ menores que 3. Outra vantagem do uso de DMSP em ambientes aquáticos envolve a capacidade desses amostradores em concentrar todos os compostos neutros hidrofóbicos com massas molares menores que $600 \mathrm{Da}$. Assim, oferecem larga faixa de aplicabilidade com relação a classe química e massa molar que não é obtida por nenhum outro amostrador.

De acordo com PETTY et al. (2000), o uso dos DMSP pode ser vantajoso para as seguintes aplicações:

- determinar fontes de poluição; 
- estimar a concentração média ao longo do tempo (CMLT);

- simular a concentração de compostos químicos biodisponíveis para testes com bioindicador ou imunoensaios;

- seqüestrar contaminantes para os procedimentos de avaliação e identificação de toxicidade (AIT); e

- estimar a exposição de organismos e a bioconcentração potencial.

Alguns projetos empregando DMSP têm sido realizados pela Agência de Proteção Ambiental dos Estados Unidos da América (EPA-US), tais como "use of long-term in situ sorbent and rapid biossays in groundwater monitoring" (GUSTAVSON, 2001).

Trabalhos têm sido reportados na literatura sobre a aplicação de DMSP para amostragem de hidrocarbonetos aromáticos policíclicos (HAP) (LEBO et al., 1992), dibenzo-p-dioxinas, dibenzofuranos e bifenilas policloradas (BPC) (LUNDGREN et al., 2003; RANTALAINEN, IKONOMOU e ROGERS, 1998; BERGQVIST, STRANDBERG e RAPPE, 1999), marcadores de combustíveis (LUELLEN e SHEA, 2003) e agrotóxicos organoclorados (ELLIS et al., 1995) em corpos d'água.

SABALIUNAS et al. (2003) calcularam as concentrações e a taxa de remoção de triclosan (bacterícida utilizado em produtos de higiene) na bacia do rio Aire (Reino Unido), usando DMSP expostos em vários locais. A estimativa da taxa de remoção calculada para a água foi inserida no programa Geography-Referenced Regional Exposure Assessment Tool for European Rivers (GREATER) para prever a distribuição da concentração de triclosan na bacia em questão, assim como para calcular sua concentração no corpo hídrico. Os resultados obtidos da taxa de remoção $\left(0,21-0,33 \mathrm{~h}^{\prime 1}\right)$ indicaram que o triclosan foi rapidamente eliminado do ambiente aquático.

VERWEIJ et al. (2004) estudaram a biodisponibilidade de HAP, BPC e compostos orgânicos persistentes (COP) em água e peixes nas proximidades da cidade de Amsterdã - Holanda. Correlação significante foi observada entre as concentrações de metabólitos de HAP analisados em peixes e água, sugerindo que o método de DMSP reproduz com exatidão a exposição de HAP em ecossistemas aquáticos.

RANTALAINEN, IKONOMOU e ROGERS (1998) estudaram os DMSP em água e sedimentos do Rio Lower Fraser no Canadá para avaliar os níveis de contaminação por dioxinas, furanos e BPC. Pesquisaram o acúmulo dos poluentes no DMSP e nos tecidos de peixes que se alimentam de espécies bentônicas. Os dados obtidos para o acúmulo em tecidos dos peixes apresentaram perfil similar aos resultados encontrados utilizando os dispositivos.

PETTY et al. (1998) utilizaram o DMSP para avaliar a presença de pesticidas organoclorados, HAP e BPC no Rio Missouri e (três) afluentes. O estudo foi realizado após enchente na região e os resultados comparados aos dados obtidos antes da enchente. Após a enchente, os contaminantes foram detectados em todos os locais em níveis mais altos que os obtidos no período anterior.

UTVIK e JOHNSEN (1999) utilizaram o DMSP e mexilhões (Mytilus edulis) para determinar a fração de HAP biodisponíveis nas proximidades de uma plataforma no Mar do Norte, na Noruega. Os resultados desse estudo indicaram que ambas as técnicas favorecem informações importantes a respeito da biodisponibilidade de HAP para organismos marinhos.

\subsection{ESPECIFICAÇÕES DO DMSP}

HUCKINS et al. (1990) propuseram um DMSP com a seguinte configuração padrão: comprimento da membrana de polietileno de $90 \mathrm{~cm}$; largura da membrana de $2,5 \mathrm{~cm}$, espessura da membrana de 75-90 $\mu \mathrm{m}$, área superficial de $450 \mathrm{~cm}^{2}$, relação entre as massas de membrana $\left(\mathrm{M}_{\mathrm{m}}\right)$ e trioleína $\left(\mathrm{M}_{\mathrm{L}}\right)$ $\mathrm{M}_{\mathrm{m}} / \mathrm{M}_{\mathrm{L}}(\mathrm{w} / \mathrm{w})$ de $4: 1$, sendo que a massa trioleína foi igual a 0,91 $\mathrm{g}(1 \mathrm{~mL})$ e a massa DMSP aproximadamente 4,6 g. Relação importante nessa configuração padrão é a área superficial/comprimento da membrana que influencia na determinação das taxas de amostragem. 


\subsection{PROCEDIMENTO PARA MANUSEIO E EXPOSIÇÃO DOS DMSP}

Os DMSP são capazes de seqüestrar grande variedade de compostos, portanto cuidados devem ser tomados durante seu manuseio e exposição para evitar que contaminações provoquem erros nas análises. Os cuidados com o manuseio incluem: acondicionamento e transporte adequados antes e após a exposição, seleção do local para exposição, instalação do dispositivo, coleta das amostras e procedimentos laboratoriais (PETTY et al., 2000).

O contato dos DMSP com o ar deve ser evitado, uma vez que pode ocorrer contaminação por compostos na fase gasosa. Os técnicos que os manuseiam devem evitar o uso de loções, perfumes, ou outros cosméticos que possam ser concentrados no dispositivo (PETTY et al., 2000).

Os DMSP devem ser acondicionados em recipientes apropriados antes e após a coleta, sendo as amostras (coletadas) armazenadas em temperaturas baixas (menores que $-15^{\circ} \mathrm{C}$ ) para a preservação até o momento da análise. Pode-se utilizar folha de alumínio para embrulhar o DMSP amostrado (PETTY et al, 2000).

O dispositivo deve ser exposto em profundidade adequada, evitando-se problemas como abaixamento do nível de água durante períodos de seca. É recomendado camuflar os dispositivos para prevenir perdas por vandalismo e utilizar mecanismos para fixa-los no local desejado. Pode-se expor o dispositivo horizontalmente ou verticalmente, conforme Figura 2 (CHAPMAN, 2002).

Durante a exposição podem ser usados vários tipos de protetores ao redor do DMSP, como os apresentados nas Figuras 2 e 3 . As superfícies metálicas (como aço) são mais apropriadas por estarem livres de potenciais interferências. ELLIS et al. (1995) recomendam a utilização de telas de cobre para redução do crescimento de microorganismos na superfície da membrana. A proteção é feita para minimizar a abrasão da membrana, principalmente em ambientes de regimes turbulentos. Deve-se expor ao máximo a superfície da membrana, mas recomenda-se protege-la da exposição direta à luz solar para evitar fotólise de alguns compostos.

FIGURA 2 - FORMAS DE EXPOSIÇÃO DOS DMSP: (A) HORIZONTAL; (B) VERTICAL

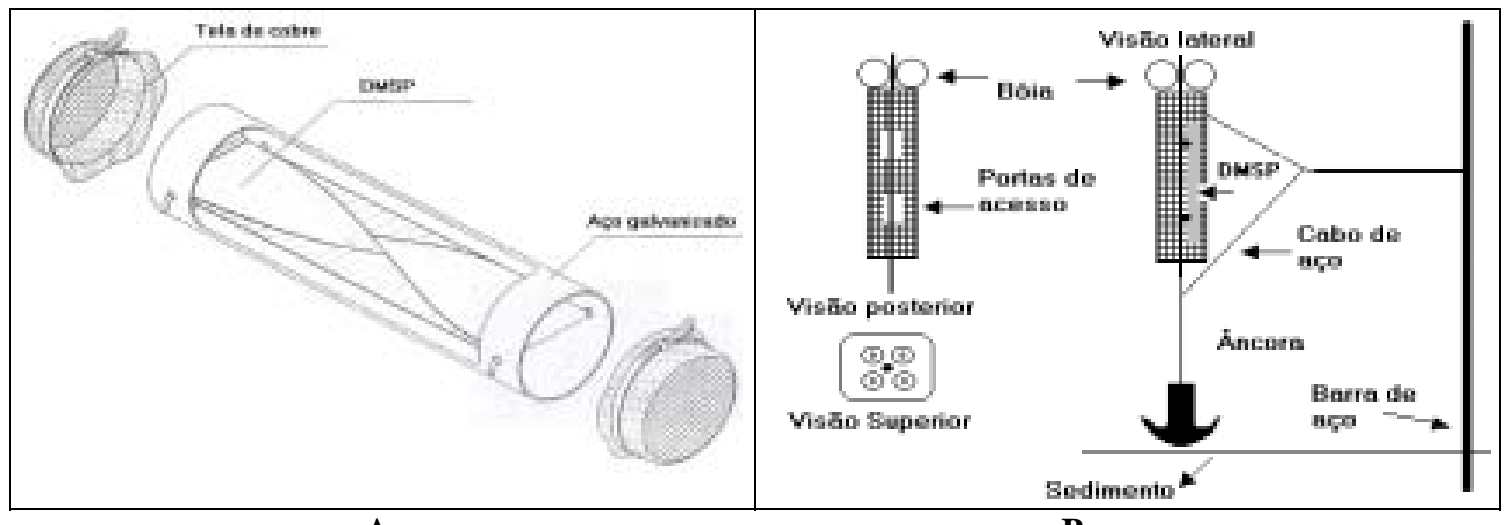

A

B

Fonte: Modificado de CHAPMAN (2002).

\subsection{PERÍODO DE EXPOSIÇÃO}

Devido à natureza integrativa do processo de amostragem, os DMSP podem ser expostos em intervalos de dias ou meses dependendo do contaminante que se deseja investigar. Geralmente tem sido utilizado período de 14 a 30 dias para seqüestrar nível quantificável para a maioria dos contaminantes hidrofóbicos de interesse ambiental (PETTY et al., 2000). 


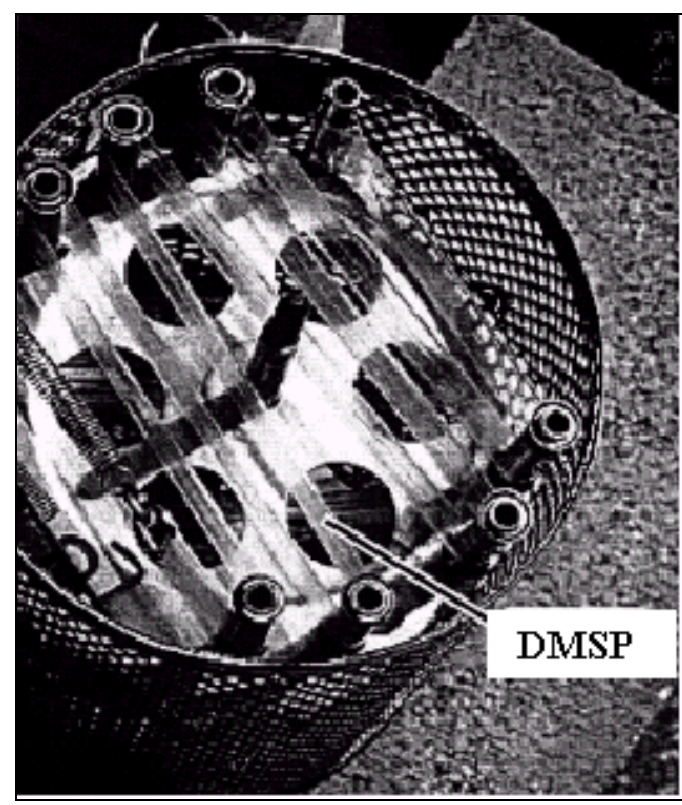

A

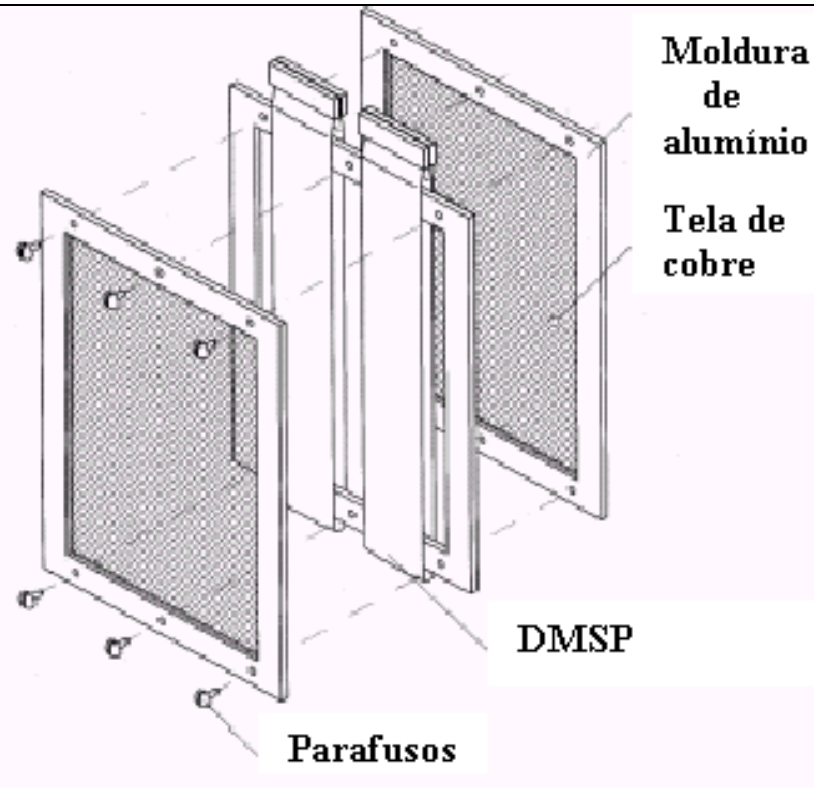

$\mathbf{B}$

Fatores como tipo de analito, limitações analíticas e variáveis ambientais do local de exposição são considerados para a seleção de intervalo apropriado para amostragem. Variáveis ambientais como temperatura, condições hidrodinâmicas e potencial de deposição de microorganismos devem ser verificadas para cada local de amostragem.

\subsection{CALIBRAÇÃO DOS AMOSTRADORES}

Os DMSP precisam ser calibrados em condições controladas de laboratório para obtenção de fator de correção, levando em consideração as possíveis interferências das condições ambientais durante o período de exposição que podem afetar o resultado da análise.

De acordo com HUCKINS et al. (1999) são utilizados compostos de referência permeável (CRP) para a calibração. Esses, são compostos orgânicos não-interferentes com moderado a alto coeficiente de partição octanol-água $\left(\mathrm{K}_{\text {ow }}\right)$, que são adicionados ao lipídio do DMSP antes de confiná-lo na membrana. As perdas desses compostos medidas durante a exposição ambiental são comparadas com os valores obtidos nos estudos de calibração em laboratório, podendo-se corrigir os efeitos das variáveis ambientais como temperatura, turbulência e deposição de microorganismos (PETTY et al., 2000; HUCKINS et al., 2002; VRANA e SCHÜÜRMANN, 2002).

\section{PROCESSAMENTO E ANÁLISE DAS AMOSTRAS}

O processamento das amostras para extração dos analitos envolve a remoção dos microrganismos, a extração ou diálise e a purificação. Na etapa de remoção de microorganismos ocorrem a lavagem do dispositivo com solvente, geralmente utilizando hexano, a limpeza com água deionizada corrente, a limpeza com escova macia para remoção da camada de microorganismos, a imersão em ácido, a lavagem com água deionizada corrente e quando necessário, limpeza com acetona e depois com isopropanol, e secagem em nitrogênio de alta pureza. As etapas de lavagem com 
solventes apolares devem ser evitadas ou minimizadas, pois podem ocorrer perdas (VRANA et al., 2001). Na etapa da extração ou diálise é preciso escolher o solvente mais adequado, sendo utilizados geralmente hexano e/ou diclorometano. A extração deve ser feita em câmara (fechada para evitar contaminação) contendo o solvente, sendo verificadas a quantidade de solvente e de extrações necessárias e o tempo de extração. Outra forma de extração é cortar o DMSP para avaliar, separadamente, a membrana de polietileno e a trioleína. Também se deve reduzir o volume de cada amostra extraída para o volume apropriado para a próxima etapa. Outras questões que podem ser avaliadas para melhoria dos resultados envolvem a otimização das condições de diálise (como tempo de extração), quantidade e tipo de solvente, otimização da configuração dos dispositivos para exposição e verificação da degradação dos analitos no meio lipídico.

A etapa da purificação pode ser efetuada por meio de cromatografia em coluna. Geralmente são utilizadas colunas de alumina ativada ou sílica gel (ZIMMERMAN, THURMAN e BASTIAN, 2000). As fases móveis mais utilizadas são hexano, diclorometano, metanol ou misturas desses solventes.

$\mathrm{Na}$ etapa da análise instrumental, geralmente, são utilizadas técnicas como Cromatografia a Gás (CG), CG acoplada com Espectrometria de Massas e Cromatografia a Líquido de Alta Eficiência (CLAE).

\section{CÁlCULO DA TAXA DE AMOSTRAGEM E CONCENTRAÇÃO DOS ANALITOS}

A estimativa da concentração média de poluentes lipofílicos em ambientes aquáticos ao longo do tempo pode ser obtida pela concentração do analito no DMSP e a utilização de modelos matemáticos.

\subsection{MÉTODO 1}

A capacidade do DMSP para acumular contaminantes orgânicos dissolvidos geralmente é relatada em termos do coeficiente de partição octanol-água $\left(\mathrm{K}_{\text {ow }}\right)$. Esse parâmetro é similar ao coeficiente de partição trioleína-água $\left(\mathrm{K}_{\mathrm{Lw}}\right)$. $\mathrm{O} \mathrm{K}_{\mathrm{ow}}$ ou $\mathrm{K}_{\mathrm{Lw}}$, as propriedades físico-químicas da membrana e das moléculas do contaminante, bem como as condições ambientais afetam a taxa de acúmulo dos poluentes no DMSP (MEADOWS et al.,1998).

Segundo VRANA e SCHÜÜRMANN (2002), a taxa de amostragem dos contaminantes da água para o DMSP deve ser descrita em termos da $2^{2}$ Lei de Fick aplicada para cada compartimento do sistema (lipídio, membrana e água próxima à superfície do amostrador). A heterogeneidade de cada fase devido a presença de camadas de difusão e as diferentes solvatações das substâncias em cada fase deviam ser consideradas, mas a descrição resultante seria muito complicada. Descrição menos complexa foi elaborada por HUCKINS et al. (1999), considerando o DMSP como compartimento simples, ao assumir a rápida difusão dentro dos compartimentos, dado o tempo de duração dos experimentos. HUCKINS et al. (1999) consideraram a membrana de PE com um volume equivalente ao da fase do lipídio (trioleína). Desta forma, o coeficiente de partição DMSP-água $\left(\mathrm{K}_{\mathrm{DMSP}}\right)$ e a concentração do analito no DMSP podem ser expressos como:

$$
K_{D M S P}=K_{L w}\left(V_{L}+K_{m L} V_{m}\right) N_{D M S P} \quad \text { Equação } 1
$$

e

$$
C_{\text {DMSP-e }}=C_{w} K_{L w}\left(V_{L}+K_{m L} V_{m}\right) / V_{D M S P} \quad \text { Equação } 2
$$

Na qual:

$$
K_{D M S P}=\text { coeficiente de partição DMSP-água; }
$$


$K_{L w}=$ coeficiente de partição lipídio-água;

$K_{m L}=$ coeficiente de partição membrana-lipídio;

$V_{L}=$ volume de lipídio;

$V_{m}=$ volume da membrana;

$V_{D M S P}=$ volume do DMSP;

$C_{\text {DMSP-e }}=$ concentração do analito no DMSP no equilíbrio;

$C_{w}=$ concentração do analito na água.

Ao assumir a aproximação de "lipídio-equivalente" e o controle da taxa de entrada na camada aquosa de difusão para o dispositivo inteiro, a concentração do analito no DMSP é dada por:

$$
C_{D M S P}=C_{w} K_{D M S P}\left\{1-\exp \left[-K_{w} A t / K_{L w}\left(V_{L}+K_{m L} V_{m}\right)\right]\right\} \quad \text { Equação } 3
$$

Na qual:

$K_{w}=$ coeficiente de transferência de massa na fronteira (camada aquosa);

$A=a ́ r e a ;$

$\mathrm{T}=$ tempo

O controle da taxa de transferência do analito apenas pela membrana é fornecido pela Equação 4.

$$
C_{D M S P}=C_{w} K_{D M S P}\left\{1-\exp \left[-K_{m} K_{m w} A t / K_{L w}\left(V_{L}+K_{m L} V_{m}\right)\right]\right\} \quad \text { Equação } 4
$$

Na qual:

$K_{m}=$ coeficiente de transferência de massa na membrana;

$K_{m w}=$ coeficiente de partição membrana-água.

A concentração do analito na água $\left(C_{w}\right)$ pode ser obtida diretamente pela simplificação das Equações 3 e 4.

$$
C_{w}=C_{D M S P} / K_{D M S P}\left[1-\exp \left(-K_{e} t\right)\right] \quad \text { Equação } 5
$$

Na qual:

$$
K_{e}=K_{w} A / K_{L w}\left(V_{L}+K_{m L} V_{m}\right) \text { ou } K_{m} K_{m w} A / K_{L w}\left(V_{L}+K_{m L} V_{m}\right) \text {. }
$$

As duas versões de $K_{e}$ representam a taxa constante de eliminação ou coeficiente de troca $\left(\mathrm{t}^{-1}\right)$ para a taxa global de eliminação. Quando $\mathrm{K}_{\mathrm{e}} \mathrm{t}$ é pequeno $(<-\ln 0,5)$ ou $\mathrm{C}_{\mathrm{DMSP}} / \mathrm{C}_{\mathrm{w}}<-\ln 0,5 \mathrm{~K}_{\mathrm{DMSP}}$, a taxa de entrada de substâncias é linear e integrativa. Na região linear da taxa, a equação acima se reduz a:

$$
C_{w}=C_{D M S P} V_{D M S P} / R_{s} t=C_{D M S P} / K_{u} t \quad \text { Equação } 6
$$

Na qual:

$R_{s}=$ volume de água removido por unidade de tempo (L/d) por DMSP padrão com $1 \mathrm{~g}$ de trioleína;

$K_{u}=$ taxa de entrada constante $(\mathrm{mL} / \mathrm{d} . \mathrm{g})$;

$\mathrm{R}_{\mathrm{s}}=$ grupos $K_{w} A$ (controle na camada da fronteira) ou $K_{m} K_{m w} A$ (controle na membrana).

A relação entre $K_{D M S P}$ e a taxa contante $K_{u}$ é dada por: 


$$
K_{D M S P}=K_{u} / K_{e}=R_{s} / K_{e} V_{D M S P}
$$

sendo que $R_{s}$ está relacionado a $K_{e}$ por:

$$
R_{s}=K_{e} K_{D M S P} V_{D M S P}
$$

Tem-se, então, a concentração do analito na água pela equação 9:

$$
\begin{array}{lr}
C_{w}=\frac{C_{S P M D} V_{S P M D}}{R_{s} t} & \text { Equação } 9 \\
\text { ou } & \text { Equação } 10
\end{array}
$$

\subsection{MÉTODO 2}

Ao utilizar o DMSP para estimar a concentração de contaminantes dissolvidos na água é necessário calibrá-lo por meio de medidas da taxa de amostragem em condições controladas. A concentração pode ser estimada usando-se a equação (LUELLEN e SHEA, 2002) :

$$
C_{w}=\frac{C_{S P M D}}{K_{1} t}
$$

Na qual:

$\mathrm{Cw}$ = concentração do analito na água (ng/L);

$\mathrm{C}_{\mathrm{DMSP}}=$ concentração do analito no DMSP $(\mathrm{ng} / \mathrm{g})$;

$\mathrm{K}_{1}=$ constante de primeira ordem $\left(\mathrm{Lg}^{-1} \mathrm{~d}^{-1}\right)$;

$\mathrm{T}=$ tempo de exposição $(\mathrm{d})$.

A Equação 11 pode ser expressa em termos do volume de água que é quantitativamente extraído pelo dispositivo por unidade de tempo, ou taxa de amostragem efetiva, $R_{s}\left(L . d^{-1}\right)$ :

$$
C_{w}=\frac{N_{S P M D}}{R_{s} t} \quad \text { Equação } 12
$$

Na qual:

$\mathrm{N}_{\text {DMSP }}=$ massa de analito acumulada no DMSP.

$R_{s}$ pode ser determinada em laboratório mantendo $C_{w}$ constante. Essa equação pode ser utilizada para determinação de $R_{s}$ de maneira mais simples que a exposta na Equação 8.

Ao utilizar o dispositivo é preciso considerar que somente a fração livremente dissolvida dos analitos está disponível para a partição na membrana. Entretanto, essa questão não tem sido avaliada rigorosamente, sendo razoável incorporar uma correção para partição em carbono orgânico dissolvido (COD) e/ou carbono orgânico particulado (COP). A Equação 12 pode ser modificada incorporando essa questão, obtendo-se a estimativa da concentração total dos contaminantes dissolvidos em água $\left(\mathrm{C}_{w}, t d\right)$ :

$$
C_{w, t d}=\left(1+C_{C O D} K_{C O D}\right) N_{D M S P} / R_{s} \cdot t \quad \text { Equação } 13
$$

Na qual:

$C_{C O D}=$ concentração de COD;

$K_{C O D}=$ coeficiente de partição dos compostos na água e COD. 
Utilizando as equações mencionadas acima pode-se estimar a concentração média de contaminantes lipofílicos ao longo do tempo em ambientes aquáticos. Assim, determinam-se as concentrações dos analitos no DMSP e as taxas de amostragem, sendo recomendado a realização de estudos de calibração dos dispositivos em laboratório.

\section{FATORES QUE INFLUENCIAM A AMOSTRAGEM E ENSAIO DOS POLUENTES}

A amostragem de contaminantes por DMSP pode ser influenciada por fatores como a configuração do amostrador e as propriedades físico-químicas das moléculas dos poluentes, além da temperatura, do crescimento de microorganismos no dispositivo, das condições hidrodinâmicas (velocidade e turbulência da água) e da salinidade (VRANA e SCHÜÜRMANN, 2002).

O crescimento de microorganismos nos dispositivos influencia a taxa de amostragem devido à barreira formada na superfície dos mesmos, que dificulta a entrada dos contaminantes no DMSP. Para minimizar a deposição de microorganismos ELLIS et al. (1995) propuseram a utilização de telas de cobre ao redor dos dispositivos, o qual é tóxico para organismos como algas.

BOOIJ, SLEIDERINK e SMEDES (1998) estudaram os efeitos da mudança da turbulência do fluxo na cinética de transferência de compostos organoclorados em sedimentos em suspensão, abrangendo larga faixa de valores de $\mathrm{K}_{\mathrm{ow}}\left(4<\log \mathrm{K}_{\mathrm{ow}}<8\right)$. Evidenciaram que a taxa média de transferência dos compostos da água para o DMSP é reduzida quatro vezes sob condições de baixa turbulência.

As mudanças na velocidade-turbulência do fluxo do meio de exposição afetam a espessura efetiva da camada externa. Como a resistência e a transferência de massa é diretamente proporcional a essa espessura, as taxas de amostragem dos analitos variam com a hidrodinâmica do local de amostragem. A cinética de transferência de compostos com log $\mathrm{K}_{\mathrm{ow}} \geq 4,4$ é controlada pela camada aquosa externa (assumindo velocidades da água na superfície da membrana do DMSP menores que $\left.1,0 \mathrm{~cm} \cdot \mathrm{s}^{-1}\right) \mathrm{e}$, assim as taxas de amostragem dos compostos podem variar de acordo com as condições hidrodinâmicas. Dentre os compostos com log $\mathrm{K}_{\mathrm{ow}} \geq 4,4$ estão incluídos quase todos os BPC, dioxinas e furanos, pesticidas organoclorados e hidrocarbonetos aromáticos policíclicos (HUCKINS et al., 2002). Para evitar a influência dessas variáveis, os DMSP têm sido calibrados com CRP, os quais estão sendo estudados almejando melhoria nos resultados obtidos com os DMSP. Outro tipo de interferência analítica pode ocorrer devido às impurezas presentes no dispositivo ou no ambiente de exposição. Alguns dos potenciais interferentes presentes no dispositivo são oligômeros de polietileno e resíduos da síntese de trioleína como o ácido óleico e o éster oleato de metila. O enxofre que pode ser encontrado nos locais de exposição também interfere nas análises. Alguns desses resíduos podem ser retirados na etapa de purificação por meio de cromatografia em coluna. Na fabricação do DMSP comercial é efetuada a purificação da trioleína para remover e/ou minimizar tais interferentes (PETTY et al., 2000).

\section{CONCLUSÃO}

A tecnologia de DMSP está sendo aplicada crescentemente para verificar a presença, as concentrações e os efeitos potenciais de misturas complexas de substâncias químicas. Em geral, as misturas de contaminantes seqüestradas por DMSP podem ser analisadas por qualquer técnica aplicável para determinação qualitativa e quantitativa de analitos particulares em outras matrizes ambientais. Os procedimentos expostos para processamento da amostra, enriquecimento do resíduo e análise podem ser aplicados para amostras coletadas em larga variedade de ambientes, possibilitando a identificação de vários cenários de poluição. A tecnologia de DMSP continua a ser pesquisa por cientistas ambientais que buscam a otimização das condições de exposição e análise. 


\begin{abstract}
MONITORING OF PESTICIDE AND OTHER ORGANIC POLLUTANTS IN WATER SOURCES BY UTILIZING SEMIPERMEABLE MEMBRANE DEVICES

A review of research works about semipermeable membrane devices (SPMD), containing triolein for aquatc environment monitoring was accomplished. The SPMD have been utilized as passive pesticide samplers and other lypophylic pollutants in water. Those devices have been efficient in sampling water for substance analysis in trace and ultra-trace levels, for organochlorine, organophosphorus pesticides, polychlorinated biphenyl, polycyclic aromatic hidrocarbons, dioxins, furans and other non polar organic substances. Special attention is given to models for sampling index calculation and concentration in water corps. The SPMD technology continues to be researched by environmental scientists that search the optimization of exposure conditions and analysis.
\end{abstract}

KEY-WORDS: SAMPLING; SPMD; PESTICIDES; WATER.

\title{
REFERÊNCIAS
}

1 ABNT. Associação Brasileira de Normas Técnicas. NBR 9897: planejamento de amostragem de efluentes líquidos e corpos receptores. Rio de Janeiro, 1987a. $23 \mathrm{p}$.

2 ABNT. Associação Brasileira de Normas Técnicas. NBR 9898: preservação e técnicas de amostragem de efluentes líquidos e corpos receptores. Rio de Janeiro, 1987b. 34 p.

3 BAIRD, C. Química ambiental. 2.ed. Porto Alegre: Bookman, 2002. 622 p.

4 BERGQVIST, P.A.; STRANDBERG, B.; RAPPE, C. Lipid removal using semipermeable (SPMs) in PCDD and PCDF analysis of fat-rich environmental samples. Chemopsphere, v. 38, n. 5, p.933-943, 1999.

5 BOOIJ, K.; SLEIDERINK, H. M.; SMEDES, F. Calibrating the uptake kinetics of semipermeable membrane devices using exposure standards. Environmental Toxicology and Chemistry, v. 17, n. 7, p. 1236-1245, 1998.

6 CHAPMAN, D. The virtual fish: SPMD basics. U.S. Geological Survey - Columbia Environmental Research Center. Disponível em: <http://wwwaux.cerc.cr.usgs.gov/spmd>. Acesso em: 15 de outubro de 2002.

7 ELLIS, G. S.; HUCKINS, J.N.; ROSTAD, C. E.; SCHIMITT, C. J.; PETTY, J. D.; MACCARTHY, P. Evaluation of LipidContaining Semipermeable Membrane Devices for Monitoring Organochorine Contaminants in the Upper Mississipi River. Environmental Toxicology and Chemistry, v. 14, n. 11, p. 1875-1884, 1995.

8 GUSTAVSON, K.L. Use of long-term in situ sorbent and rapid biossays in groundwater monitoring. [Washington]: EPA, Project number U915464, 2001.

9 HUCKINS, J.N.; TUBERGEN, M. W.; MANUWEERA, G.K. Semipermeable membrane devices containing model lipid: a new approach to monitoring the biovalilability of lipophilic contaminants and estimating their bioconcentration potencial. Chemosphere, v. 20, n. 5, p. 533-552, 1990.

10 HUCKINS, J. N.; PETTY, J. D.; ORAZIO, C. E.; LEBO, J. A.; CLARK, R. C.; GIBSON, V. L.; GALA, W. R.; ECHOLS, K. R. Determination of uptake kinetics (sampling rates) by lipid-containing semipermeable membrane devices (SPMDs) for polycyclic aromatic hydrocarbons (PAHs) in water. Environ. Sci. Technol., v. 33, p. 3918-3923, 1999.

11 HUCKINS, J. N.; PETTY, J. D.; LEBO, J. A.; ALMEIDA, F. V.; BOOIJ, K.; ALVAREZ, D. A.; CRANOR, W. L.; CLARK, R. C.; MOGENSEN, B. B. Development of permeability reference compound approach for in situ calibration of semipermeable membrane devices. Environ. Sci. Technol., v. 36, p. 85-91, 2002.

12 KOT, A.; ZABIEGATA, B.; NAMIESNIK, J. Passive sampling for long-term monitoring of organic pollutants in water. Trends in Analytical Chemistry, v.9, p.446-459, 2000.

13 LEBO, J.A.; ZAJICEK, J.L.; HUCKINS, J.N.; PETTY, J.D.; PETERMAN, P.H. Use of semipermeable membrane devices for in situ monitoring of polycyclic aromatic hydrocarbons in aquatic environments. Chemosphere, v. 25, n. 5, p.697-718, 1992.

14 LEBO, J. A.; GALE, R. W.; PETTY, J. D.; TILLITT, D. E.; HUCKINS, J. N.; MEADOWS, J. C.; ORAZIO, C. E.; ECHOLS, K. R.; SCHRORDER, D. J. Use of semipermeable membrane devices as an in situ sampler of waterborne bioavailable PCDD and PCDF residue at sub-parts-per-quadrillion concentrations. Environ. Sci. Technol., v. 29, p. 2886-2892, 1995.

15 LU, Y.; WANG, Z. Accumulation of organochlorinated pesticides by triolein-containing semipermeable membrane device (triolein-SPMD) and rainbow trout. Water Research, v.37, n.10, p. 2419-2425, 2003.

16 LUELLEN, D. R., SHEA, D. Calibration and field verification of semipermeable membrane devices for measuring 
polycyclic aromatic hydrocarbons in water. Environ. Sci. Technol., v. 36, p. 1791-1797, 2002.

17 LUELLEN, D. R.; SHEA, D. Semipermeable membrane devices accumulate conserved ratios of sterane and hopane petroleum biomarkers. Chemosphere, v. 53, n.7, p. 705-713, 2003.

18 LUNDGREN, K.; TYSKLINDA, M.; ISHAQB, R.; BROMANB, D.; BAVELC, B. V. Flux estimates and sedimentation of polychlorinated naphthalenes in the northern part of the baltic sea. Environmental Pollution, v.126, n. 1, p.93105, 2003.

19 MAGDIC, S.; PAWLISZYN, J. B. Analysis of organochlorine pesticides using solid-phase microextration. Journal of Chromatography A, v. 723, p. 111-122, 1996.

20 MEADOWS, J. C.; ECHOLS, K. R.; HUCINS, J. N.; BORSUK, F. A.; CARLINE, R. F.; TILLITT, D. E. Estimation of uptake rate constants for PCB congeners accumulated by semipermeable membrane devices and brown trout (Salmo trutta). Environ. Sci. Technol., v. 32, p. 1847-1852, 1998.

21 PETTY, J. D.; HUCKINS, J. N.; ORAZIO, C. E.; LEBO, J. A.; POULTON, B. C.; GALE, R. W.; CHARBONNEAU, C. S.; KAISER, E. M. Determination of waterborne bioavailable organochlorine pesticide residues in the lower missouri river. Environ. Sci. Technol., v. 29, p. 2561-2566, 1995.

22 PETTY, J. D.; POULTON, B. C.; CHARBONNEAU, C. S.; HUCKINS, J. N.; JONES, S. B.; CAMERON, J. T.; PREST, H. F. Determination of bioavailable contaminants in the lower Missouri River following the flood of 1993. Environ. Sci. Technol., v. 32, p. 837-842, 1998.

23 PETTY, J. D.; ORAZIO, C. E.; HUCKINS, J. N.; GALE, R. W.; LEBO, J. A.; MEADOWS, J. C.; ECHOLS, K. R.; CRANOR, W. L. Considerations involved with the use of semipermeable membrane devices for monitoring environmental contaminants. Journal of Chromatography A., v. 879, p. 83-95, 2000.

24 RANTALAINEN, A.; IKONOMOU, M.G.; ROGERS, I.H. Lipid-containing semipermeable membrane devices (SPMD) as concentrators of toxic chemicals in the lower fraser River, Vancouver, British Columbia. Chemosphere, v. 37, n. 6, p. 1119-1138, 1998.

25 SABALIUNAS, D.; WEBBB, S. F.; HAUKC, A.; JACOBC, M.; ECKHOFF, W. S. Environmental fate of Triclosan in the River Aire Basin, UK. Water Research, v.37, n.13, p. 3145-3154, 2003.

26 UTVIK, T.I.R.; JOHNSEN, S. Bioavailability of polycyclic aromatic hydrocarbons in north sea. Environ. Sci. Technol., v. 33, p. $1963-1969,1999$.

27 VERWEIJ, F.; BOOIJB, K.; SATUMALAYA, K.; MOLENA, N. D.; OOSTA, R. D. Assessment of bioavailable PAH, PCB and OCP concentrations in water, using semipermeable membrane devices (SPMD), sediments and caged carp. Chemosphere., v.54, n.11, p.1675-1689, 2004.

28 VRANA, B.; PASCHKE, A.; POPP, P.; SCHÜÜRMAN, G. Use of semipermeable membrane devices (SPMD): determination of bioavailable, organic, waterborne contaminants in the industrial region of bitterfeld, saxony-anhalt, Germany. Environ. Sci. \& Pollut. Res., v. 8, n.1, p. 27-34, 2001.

29 VRANA, B., SCHÜÜRMAN, G. Calibrating the uptake kinetics of semipermeable membrane devices in water: impact of hydrodinamics. Environ. Sci. Technol., v. 36, p. 290-296, 2002.

30 ZIMMERMAN, Z. R.; THURMAN, E.M.; BASTIAN, K.C. Detection of persistent organic pollutants in the Mississippi Delta using semipermeable membrane devices. Science of the Total Environment, v. 248, p. 169-179, 2000. 